\title{
Classer la femme séropositive Ce qu'elle est, ce qu'elle fait, qui elle fréquente
}

\author{
Marie-Ange SCHILTZ ${ }^{1}$
}

Les travaux de Erving Goffman et lan Hacking sur la construction des faits sociaux et les attentes normatives que ces opérations engendrent $[1,2]$ ne nous permettent plus de négliger la part de présupposés sociaux et éventuellement moraux, qui participent à l'élaboration des catégories utilisées dans la connaissance du social. Ils ne nous permettent plus également d'ignorer leurs effets sur la perception que la société a sur les individus ainsi étiquetés, ni sur celle que ces individus ont sur eux-mêmes.

En s'appuyant sur les pratiques de classement des personnes atteintes par le VIH actuellement en vigueur en épidémiologie, ce chapitre propose d'examiner l'assignation des femmes séropositives de l'enquête Anrs-Vespa [3].

Sur la base de comportements dits à risque ou d'un environnement social à forte prévalence du VIH, l'épidémiologie a produit des délimitations et des informations sur des groupes de population considérés comme plus exposés au risque de transmission par le VIH.

Au moment des premières définitions du sida, alors que les vecteurs de la transmission étaient encore inconnus, la construction de "groupes à risque " à partir des seules observations statistiques a été un enjeu de lutte de la part des personnes désignées comme victimes potentielles [4]. En effet, dans ce contexte d'ignorance médicale, apparaît un trop plein d'hypothèses, souvent empreint de présupposés moraux, mettant en cause, de façon parfois contradictoire, les modifications des contacts entre populations, la multiplication des échanges, l'environnement et les styles de vie. Les homosexuels masculins avec, dans une moindre mesure, les toxicomanes par voie intraveineuse et les Haïtiens, groupes déjà stigmatisés où se sont concentré les premiers cas de déficit immunitaire enregistrés, ont le sentiment d'être à nouveau dénoncés pour leur façon de vivre, sans pouvoir mettre en œuvre des stratégies de protection contre le danger létal qui les menace. Par la suite, les découvertes scientifiques de l'origine virale de la maladie et de sa transmission par les liquides corporels ont permis d'éliminer quelques-unes des catégories jusqu'alors en usage et d'en asseoir d'autres. La primauté désormais accordée au virus et

CAMS-CNRS, UMR 8169, CERMES, Villejuif. les progrès dans le contrôle médical des transfusions sanguines affaiblissent l'importance des facteurs écologiques et sociaux: les catégories " Haïtien » et " hémophile » disparaissent, l'idée de " situations à risque " cherche à se substituer à celle de " groupes à risque ». A contre-courant de ces efforts de redéfinition qui s'opèrent dans un souci de suppression d'attributs négatifs, par glissement sémantique, la catégorie " contamination lors de contacts sexuels entre personnes de sexe différents " construit une nouvelle entité, la " population hétérosexuelle " qui, par effet de halo, renvoie à la catégorie "population générale " [5]. Vers la fin des années 1990, l'information sur l'origine géographique des cas hétérosexuels a cassé un peu cette globalité. La notion de zones où l'épidémie sévit de façon endémique est réactivée avec les catégories " personne originaire de l'Afrique sub-saharienne " en France métropolitaine et "personne d'origine haïtienne » dans les départements français d'Amérique [6]. Mais à aucun moment, les divers réaménagements opérés en fonction de l'évolution des connaissances médicales et de la dynamique de l'épidémie n'ont été repensés à la lumière des cas féminins et des variations du sex ratio et ce, malgré l'apparition rapide de cas de contamination féminine prouvant la plus forte probabilité de transmission de l'homme vers la femme et rendant compte, année après année, de la féminisation progressive de l'épidémie. Les alertes lancées par les associations et les chercheurs, qui ont dénoncé une épidémie invisible parmi les femmes et les méfaits non contrôlables d'un classement inadéquat, ont été également inopérantes $[7,8]$.

Jusqu'à présent, la construction épidémiologique des groupes de transmission établis à partir de cas majoritairement masculins n'a faitl'objet d'aucune révision. Or, selon Irène Théry [9], si le classement dans des groupes peut être stigmatisant pour ceux qui sont ainsi étiquetés, paradoxalement, dans le cadre de la contamination par le VIH, « le fait d'être hors groupe à risque alimente le soupçon d'un comportement individuel transgressif, surtout dans le contexte contraignant des représentations sociales de la féminité ». En référence à des représentations qui supposent un corps sain et désirable, qui doit cependant rester " un bien rare sur le plan sexuel », le corps contaminé de la femme devient suspect : " une femme ordinaire ne peut être séropositive ». Ce 
double stigmate rend difficile, si ce n'est impossible, la reconnaissance et la prise en charge collective, qui favorisent l'élaboration d'actions préventives ciblées et l'adaptation individuelle. À ces limites sexuées s'ajoutent, selon Alain Giami [10], celles qu'impose l'usage de la catégorie " hétérosexuelle " à la représentation de l'épidémie et à la capacité du groupe ainsi désigné à définir les risques encourus et la façon de les éviter.

\section{Le classement selon les « modes de transmission " établis}

Dans l'enquête Anrs-Vespa (pour la description de la méthodologie de l'enquête, voir l'encadré page 215), le classement des répondants selon la forme légitimée du « mode de transmission " utilisé en épidémiologie est effectué à partir des trois sources disponibles que sont la réponse du patient, le registre médical et le questionnaire médical ${ }^{2}$ (tableau 1).

\section{Tableau 1}

Répartition des patients infectés par le VIH selon les modes de transmission établis [11]

\begin{tabular}{lcr}
\hline \multicolumn{1}{c}{ Mode de transmission } & $\begin{array}{c}\text { Hommes } \\
\mathbf{n = 2 1 2 9}\end{array}$ & $\begin{array}{r}\text { Femmes } \\
\mathbf{n = 7 9 4}\end{array}$ \\
\hline Rapports homosexuels & $56,5 \%$ & $0,2 \%$ \\
Injection de drogue & $17,4 \%$ & $16,5 \%$ \\
Rapports hétérosexuels & $20,8 \%$ & $76,7 \%$ \\
Autres (transfusion, accident professionnel) & $2,7 \%$ & $5,6 \%$ \\
Non renseigné & $2,5 \%$ & $0,9 \%$ \\
\hline Total & $71,2 \%$ & $28,8 \%$ \\
\hline
\end{tabular}

Selon cette catégorisation, $22,3 \%$ des femmes - contre $76,6 \%$ des hommes - sont affectées à des groupes de transmission constitués sur la base de comportements statistiquement rares au niveau de la population générale qui peuvent renvoyer à des milieux spécifiques (rapport homosexuel, injection de drogue ou accident professionnel). II en ressort qu'en dépit de l'exhortation des experts à la prise en compte de l'hétérogénéité de la population féminine atteinte par le VIH [11], 76,7 \% des femmes (contre 20,8\% des hommes) se retrouvent classées dans le mode de transmission " rapports hétérosexuels ", comportement par excellence non discriminant car très majoritaire ; selon les résultats de l'enquête représentative CSF [12], $96 \%$ des personnes de 18 à 49 ans déclarent des rapports exclusivement hétérosexuels sur la vie.

Cette catégorie, qui englobe la presque totalité de la population de référence et concerne plus des trois quarts de la population considérée, produit une information socialement peu utile. II faut donc, de ce fait, se poser la question de sa pertinence.

\footnotetext{
En cas d'incohérence entre les réponses issues des trois sources, le recodage privilégie la source médicale.
}

\section{Décomposition de la catégorie " mode de transmission par rapports hétérosexuels"}

Parmi les femmes affectées à la catégorie " mode de transmission par rapports hétérosexuels ", un tri effectué sur la base de déclarations de comportements ou de modes de vie réputés à risque du VIH n'opère qu'un reclassement très partiel ${ }^{3}$. Rapporté à l'ensemble des femmes de l'étude Vespa, rares, en effet, sont celles qui, classées en mode de contamination par rapport hétérosexuels, ont déclaré avoir obtenu de l'argent ou de la drogue en échange de rapports sexuels $(2,9 \%$ des femmes contre $0,2 \%$ parmi les hommes), s'injecter ou s'être injecté de la drogue au cours de leur vie $(3,1 \%$ contre $0,8 \%$ chez les hommes) ou encore, par extension du classement en vigueur chez les hommes, avoir eu au moins un rapport homosexuel au cours de la vie $(4,5 \%$ contre $1,8 \%$ chez les hommes).

En revanche, la situation s'éclaircit lorsque l'on s'intéresse non plus aux comportements de l'individu mais à la prévalence du VIH dans son environnement géographique et social : des taux élevés de personnes contaminées dans une zone ou un groupe déterminent une origine géographique ou des partenaires sexuels « à risque $"$.

Si I'on considère les femmes originaires d'Afrique sub-saharienne, catégorie désormais en usage, on peut reclasser plus précisément $23 \%$ des femmes auparavant étiquetées du seul attribut « rapports hétérosexuels » (contre 5,6\% des hommes).

Sachant qu'à côté de la promotion du préservatif, la prévention de l'infection à VIH envisage une "meilleure " sélection des partenaires sexuels comme une stratégie recevable pour diminuer le risque individuel de transmission du virus [10], à l'inverse, déclarer un partenaire issu d'un groupe à forte prévalence du VIH peut être, dans cette logique, considéré comme une situation qui, statistiquement, augmente le risque du VIH. C'est dans cet esprit que nous avons retenu quelques indicateurs de l'enquête Vespa qui permettent de repérer l'existence, à certains moments de la vie, d'un contexte sexuel potentiellement plus contaminant. Ainsi, " savoir que son partenaire était séropositif avant de se découvrir soi-même contaminé " $(14,2 \%$ des femmes contre $3,7 \%$ des hommes), " être assuré d'avoir eu un partenaire sexuel qui est ou a été toxicomane par voie intraveineuse " $(18,1 \%$ des femmes contre $1,7 \%$ des hommes) ou " avoir un conjoint originaire d'Afrique sub-saharienne " $7,0 \%$ des femmes contre $2,6 \%$ des hommes). Par ailleurs, selon la logique probabiliste, la multiplication des expériences augmente la possibilité de rencontrer un

Selon l'usage établi, nous avons pris en compte les modes de transmission par rapports homosexuels, injection de drogue et contamination sanguine. Les rêgles de classement privilégient systématiquement ces modes de transmission particuliers par rapport au mode de transmission * rapport hétérosexuel ", lorsque plus d'un comportement * ả risque * est mentionnè. 
partenaire sexuel contaminé ${ }^{4}$. C'est dans cette optique d'exposition au risque plus élevée que nous avons pris en considération la catégorie des individus classés dans le groupe de transmission hétérosexuel déclarant plus de 20 partenaires sexuels sur la vie et, ici encore, la proportion de femmes ainsi reclassées est plus importante $(9,9 \%$ contre $7,6 \%$ parmi les hommes).

\section{Le classement de l'ensemble des femmes séropositives}

Reprenons maintenant l'examen de ce classement au regard des indicateurs biographiques que nous venons d'examiner, en les regroupant selon les rubriques suivantes (tableau 2) : au moins un comportement dit à risque d'infection à VIH (rapports homosexuels ${ }^{5}$ et injection de drogue), une origine géographique où l'épidémie sévit de façon endémique, une plus grande probabilité de rencontrer un partenaire porteur du VIH.

Tableau 2

Classement des patients selon des indicateurs biographiques

\begin{tabular}{lrr}
\hline \multicolumn{1}{c}{ Mode de transmission } & Hommes & Femmes \\
\hline Homosexuel & $56,5 \%$ & $0,2 \%$ \\
Usager de drogue par voie intraveineuse & $17,4 \%$ & $16,5 \%$ \\
Sang, accidents professionnels & $2,7 \%$ & $5,6 \%$ \\
Hétérosexuel et comportement à risque & $2,5 \%$ & $5,5 \%$ \\
Hétérosexuel et originaire d'Afrique sub-saharienne & $5,4 \%$ & $21,3 \%$ \\
Hétérosexuel et partenaire séropositif, usager de drogue & & \\
en IV ou originaire d'Afrique sub-saharienne & $6,5 \%$ & $24,9 \%$ \\
Hétérosexuel sans autre classement & $6,3 \%$ & $25,1 \%$ \\
Indéterminé & $2,5 \%$ & $0,9 \%$ \\
\hline Total & $100 \%$ & $100 \%$ \\
\hline
\end{tabular}

Malgré nos efforts de reclassement, reste que seule une minorité de femmes séropositives $(27,8 \%)$ peut être décrite sur la base d'un des comportements ou mode de vie de la personne usuellement pris en compte dans la problématique du VIH. En revanche, c'est une majorité d'hommes qui peut être ainsi désignée $(79,1 \%)$. II est possible, à ce niveau de l'analyse, de conclure que, pour les femmes, les classements conçus sur la base de critères comportementaux déviants ou d'événements médicaux accidentels rares sont peu performants.

À l'opposé, se trouvent les "laissées pour compte ", qui échappent aux tentatives d'assignation opérées à travers les indicateurs de comportement, de situation sociale ou de réseau de socialisation, que nous avons retenus parce qu'ils ont été mobilisés à

\footnotetext{
4 Reste à établir ce que peut être un * grand nombre * de partenaires sur la vie. Au vu des décroissances des taux dans l'enquête Anrs-Vespa et de la rareté des effectifs dans les enquêtes en population générale, nous avons situé ce * trop * au seuil de plus de 20 partenaires sur la vie.

5 La transmission par rapports homosexuels n'étant attestée que parmi les hommes, la déclaration d'une expérience homosexuelle au cours de la vie, repérée dans d'autres parties du questionnaire, n'entre dans les comptes que pour les comportements à risque des hommes et est exclue des comptes pour les femmes.
}

un moment ou à un autre de la recherche sur le VIH. Rapporté à l'ensemble des personnes atteintes de l'enquête Anrs-Vespa, il reste $25,1 \%$ des femmes et $6,3 \%$ des hommes pour lesquels nous n'avons d'autre information que leur comportement hétérosexuel.

Entre ces deux extrêmes (un classement selon les comportements ou les modes de vie désignés comme à risque de VIH par la pratique de l'épidémiologie et un classement peu instructif), apparait l'importance des facteurs environnementaux. La prise en compte de l'origine géographique ou des caractéristiques des partenaires sexuels permet de reclasser presque la moitié des femmes séropositives qui jusqu'alors n'avaient qu'un attribut, celui d'avoir eu dans leur vie des rapports hétérosexuels (respectivement $21,3 \%$ et $24,9 \%$, contre respectivement $5,4 \%$ et $6,5 \%$ pour les hommes).

\section{Conclusion}

Cette analyse confirme qu'une majorité de femmes séropositives échappe à la logique des classements actuellement en vigueur. Malgré une contestation qui s'est exprimée tôt et qui s'est ensuite renouvelée sans être réellement entendue, ces classements restent imprégnés des premières observations épidémiologiques. Dans la lignée des travaux de lan Hacking [2], qui ont montré le rôle primordial des catégories de perception dans la construction du social, l'examen critique auquel nous avons procédé fournit quelques pistes pour reconsidérer les catégories en usage, afin de mieux cibler les femmes exposées au risque du VIH. Dans un contexte d'inégalité des rapports hommes/femmes et de plus grande vulnérabilité économique et sociale, il apparaît que la population des femmes atteintes est moins définie par ses comportements que par le contexte d'exercice de sa sexualité. Sans action directe sur l'usage du préservatif, principal outil de la prévention, et dans le cadre d'une possible dépendance à l'homme, il est apparu qu'une forte minorité de femmes reste uniquement définie par son hétérosexualité et que lorsqu'une information supplémentaire peut être associée, elle renvoie sans autre facteur de risque individuel à un contexte de forte prévalence du VIH jusqu'à présent imparfaitement pris en charge par la prévention : souvent, ces femmes sont nées au « mauvais " endroit ou ont croisé un « mauvais » partenaire.

Comment constituer en objet sociologique cette majorité qui se dérobe aux classements en vigueur? Comment redonner une existence sociale adéquate et intelligible à toutes ces femmes hétérosexuelles que les catégories actuelles n'arrivent pas à distinguer et, de ce fait, englobent dans des désignations stigmatisantes? C'est le défi de cet ouvrage. 


\section{Références bibliographiques}

1. Goffman E. Stigmate. Les usages sociaux des handicaps, Paris : Editions de Minuit, 1975 (Le sens commun).

2. Hacking I. Entre science et réalité : la construction sociale de quoi ? Paris: Editions La Découverte, 2001 (Textes à l'appui : anthropologie des sciences et des techniques).

3. Lert F, Obadia Y et l'équipe de l'enquête Vespa. Comment vit-on en France avec le VIH/sida? Population et Société $2004 ; 406: 4$.

4. Pollak M, Schiltz MA. Identité sociale et gestion d'un risque de santé: les homosexuels face au sida. Actes de la recherche en sciences sociales 1987 ; $68: 77-103$.

5. Giami A. Les logiques et les limites de la construction épidémiologique des groupes à risque. 2002, non publié.

6. Bouillon K, Lert F, Michelot F, Schmaus A, Spire B, Dray-Spira R. Les patients vivant avec le VIH-sida dans les départements français d'Amérique : résultats de l'enquête Anrs-Vespa, 2003. Bulletin épidémiologique hebdomadaire. Numéro thématique. Infection VIH-sida en France : vision d'ensemble et spécificités des départements français d'Amérique. BEH, 2005, 46-47 : 229-44.
7. Direction générale de la santé, Sida Info Service et le Centre européen de surveillance épidémiologique du sida. Femmes et infection à VIH en Europe. Actes du colloque 14/15 novembre 1997, Paris, CFES, 1999, 331 p.

8. Act-Up-Paris. Les femmes et le VIH. Les oubliées de la recherche. Réunion publique d'information d'Act-Up, Rèunion publique d'information (RéPI), 28 novembre 2001.

9. Théry I. " Une femme comme les autres " : séropositivité, sexualité et féminité. In Séropositivité, vie sexuelle et risque de transmission du VIH, France Lert, Yves Souteyrand, Eds. Paris : Anrs, 1999, p. 113-136.

10. Giami A, Schiltz MA. 'Representation of sexuality and relations between partners. Sex research in France in the Era of AIDS. Annual Review of Sex Research 1996 ; VII : 125-157.

11. Heard I et al. Femmes et infection à VIH. Sous la direction de Jean-François Delfraissy. Prise en charge des personnes infectées par le VIH. Rapport 2002. Paris : Flammarion Médecine-Sciences, p. 289-302.

12. Bajos N, Bozon M, Beltzer N et l'équipe CSF. Contexte de la sexualité en France. Dossier de presse : premiers résultats de l'enquête CSF. Paris, Anrs, Inserm, Ined, 13 mars 2007 (www.inserm.fr). 